\section{FANCM and breast cancer risk}

Mutations in several genes, including components of the Fanconi anemia DNA repair pathway, have been implicated in familial breast cancer risk, but much of the underlying genetic architecture remains unknown. A study by Heli Nevanlinna and colleagues (Proc. Natl. Acad. Sci. USA doi:10.1073/pnas.1407909111; 6 October 2014) now reports the discovery of an association between a variant in FANCM and breast cancer risk in the Finnish population. To identify new susceptibility genes, the authors performed exome sequencing on 24 patients with breast cancer from 11 Finnish families without mutations in BRCA1 or BRCA2. After applying several filters, they further prioritized variants in DNA repair genes and genotyped the most promising candidate risk variants in two large collections of breast cancer cases and controls from the Helsinki and Tampere regions of Finland. In a combined analysis of all data sets, they identified a nonsense variant in FANCM (encoding p.GIn1701*) that was associated with a two-fold increase in risk of breast cancer $(P=0.0018)$. This variant shows strongest association with triple-negative breast cancer and is present at a frequency of $1-2 \%$ in the Finnish population. Although the variant is rare in other populations, this finding suggests that distinct FANCM variants might contribute to breast cancer risk in different populations.

\section{HIV-1 origins and spread}

Although 13 cases of cross-species transmission of a simian immunodeficiency virus (SIV) into humans have been reported, only HIV-1 group $\mathrm{M}$ has led to a pandemic. Oliver Pybus, Philippe Lemey and colleagues now report a phylogenomic analysis of HIV-1 sequences isolated from central Africa, providing insights into what distinguished the origins and spread of HIV-1 group M (Science 346, 56-61, 2014). They examined the sequences of $348 \mathrm{HIV}-1$ group M viruses from the Democratic Republic of Congo (DRC) as well as 466 viruses from neighboring countries, obtained from the Los Alamos HIV Sequence Database. Their phylogenetic analysis estimates the time of the most recent common ancestor of HIV-1 group M sequences to be approximately 1920, confirming previous studies. They further localized the spatial origin of group M to Kinshasa in the DRC, from which virus spread to the southern DRC locations of Lubumbashi and Mbuji-Mayi, both mining towns that are connected by rail routes that likely facilitated dissemination. HIV-1 group O, which has remained non-pandemic and localized to west-central Africa, emerged at a similar time and showed similar growth rates until 1960, when the growth rate of group M viruses nearly tripled. The authors suggest that changes in health care practices might have influenced the increased spread of group M, but further studies are needed to characterize what distinguished the epidemiological courses of these HIV-1 lineages.

$O B$

\section{Kabuki syndrome and HDAC inhibitors}

Heterozygous loss-of-function mutations in KMT2D cause Kabuki syndrome, which is characterized by multiple malformations and intellectual disability. KMT2D (also known as MLL2) is a histone methyltransferase that targets lysine 4 of histone $\mathrm{H} 3$ (H3K4) to promote an open chromatin state. Mice with a heterozygous loss-of-function mutation in Kmt2d are known to have hippocampal memory defects. Now, Hans Bjornsson and colleagues characterize a new mouse

Written by Orli Bahcall, Brooke LaFlamme, Emily Niemitz \& Kyle Vogan model of Kmt2d haploinsufficiency and test the potential effectiveness of histone deacetylase inhibitor (HDACi) therapy (Sci. Transl. Med. 6, 256ra135, 2014). The authors show that $K m+2 d^{+/ \beta G e o}$ mice have hippocampal memory defects and reduced neurogenesis, with loss of H3K4 trimethylation in the dentate gyrus granule cell layer of the hippocampus. They find that HDAC inhibitors, which promote an open chromatin state, increase $\mathrm{H} 3 \mathrm{~K} 4$ trimethylation in vitro in $K m+2 d^{+/ \beta G e o}$ cells and that treatment of $K m t 2 d^{+/ \beta G e o}$ adult mice with the HDAC inhibitor AR-42 for 2 weeks corrects genome-wide H3K4 trimethylation in the granule cell layer to normal levels. Interestingly, AR-42 treatment improved measures of hippocampal memory in both $K m+2 d^{+/+}$and $K m t 2 d^{+/ \beta G e o}$ mice. This work suggests that some of the neurological deficits due to KMT2D haploinsufficiency may be treatable with postnatal HDAC inhibitor therapy.

EN

\section{Flies point to human disease genes}

Michael Wangler, Hugo Bellen and colleagues report the generation of 5,857 fly stocks with ethyl methanesulfonate (EMS)-induced recessive lethal mutations on the X chromosome (Cell 159, 200-214, 2014). The authors screened mutants for defects in the nervous system, as well as phenotypes affecting pigmentation, wing veins and wing notching, as these have previously been linked to neuronal pathways. They were able to map 614 mutations to 165 genes using a combination of complementation testing and whole-genome sequencing. The authors integrated this data set with whole-exome sequence data for 1,929 individuals from families with undiagnosed mendelian diseases. As a proof-of-principle study, they followed up on six families with mutations in three genes with neuronal phenotypes in fly: DNM2, CRX and ANKLE2. Variants in DNM2 cause Charcot-Marie-Tooth neuropathy type $2 \mathrm{M}$ and were identified in two families with clinical phenotypes consistent with this disease. CRX variants were identified in three unrelated patients with Bull's eye maculopathy, expanding the known clinical manifestations of CRX mutations. Finally, ANKLE2 was identified as a novel candidate disease gene in a family with microcephaly. Further functional studies of the CRX and ANKLE2 mutations in Drosophila showed similarities to the human phenotypes and identified potential molecular mechanisms for the pathogenicity of these alleles.

$B L$

\section{GAPLINC and gastric cancer}

Jing-Yuan Fang, Jie Xu and colleagues report the identification of a long noncoding RNA (IncRNA) involved in gastric cancer tumor progression (Cancer Res. doi:10.1158/0008-5472.CAN-140686; 2 October 2014). The authors analyzed the mRNA and IncRNA transcriptional profiles of 10 pairs of gastric cancer tumor and normal samples and identified 659 significantly upregulated and 709 downregulated IncRNAs in tumors. One upregulated IncRNA, uc002kmd.1, was selected for further study. Ectopic expression of mutant p53 (Arg248Trp) led to increased expression of uc002kmd. 1 (renamed GAPLINC, for gastric adenocarcinoma predictive long intergenic noncoding RNA), and ChIP assays confirmed physical binding. Upregulation of GAPLINC was associated with shorter survival time in a set of 90 patients with gastric cancer. Further mechanistic testing of GAPLINC showed that this IncRNA has a key role in the proliferation and invasion of gastric cancer cells, with its effects mediated via the upregulation of CD44 expression. The results suggest that GAPLINC acts as a microRNA 'sponge' by competing with CD44 mRNA for binding to miR-211-3p. 